www.jmscr.igmpublication.org

Impact Factor 5.244

Index Copernicus Value: 83.27

ISSN (e)-2347-176x ISSN (p) 2455-0450

crossref DOI:_http://dx.doi.org/10.18535/jmscr/v4i7.57

Journal Of Medical Science And Clinical Research

IGM Publication

An official Publication of IGM Publication

\title{
Study on Clinical Presentation of Factor Deficient Patients Presenting to a Tertiary Care Centre of North East India
}

\author{
Authors

\section{Dr Anupam Dutta ${ }^{1}$, Dr Taniya Sarkar Dutta ${ }^{2}$, Dr Swarop Kar ${ }^{3}$, Dr Sanjeeb Kakati ${ }^{4}$ Dr Pritikar Dowerah ${ }^{5}$} \\ ${ }^{1}$ Assistant Professor of Medicine, ${ }^{2}$ Registrar of Paediatrics, ${ }^{3}$ Registrar of Medicine, \\ ${ }^{4}$ Professor of Medicine, ${ }^{5}$ Professor of Paediatrics \\ Department of Medicine and Paediatrics, Assam Medical College and Hospital, Dibrugarh \\ Corresponding Author
}

Dr Anupam Dutta

Assistant Professor of Medicine, Assam Medical College and Hospital

Revti house, PN Road, Shantipara, Dibrugarh,786001, Assam, India

Email:dranupamdutta80@gmail.com,dranupamdutta@yahoo.com

\begin{abstract}
Introduction: Haemophilia is an X-linked congenital bleeding disorder caused by coagulation factor deficiency that may present with bleeding episodes which may range from minor to severe life threatening. Factor replacement is ideal treatment but very costly and affordable only with proper government or any society funding. Aims and Objectives: We studied all the patients with factor deficiency who came to Assam Medical College and Hospital, Dibrugarh for one year from 1/7/14 to 31/6/15 with respect to their clinical presentation, severity, treatment and outcome. As factors are now available in government medical college free of cost to haemophilia patients, we were also trying to identify a subgroup of patients who could benefit from prophylactic factor.

Materials and Methods: A detailed clinical and family history was taken; proper general examination was done. Adequate laboratory investigations were done. Untested siblings were counselled and tested for factor deficiency. Factors were given to indicated patients with mild involvement as out-patients department (OPD) basis, but more severe cases were hospitalized. Proper clinical examination, routine blood tests and physiotherapy were advised in hospitalized patients.
\end{abstract}

Results and Observations: Forty five (45) cases were studied during this one year period of which, 43 were males and 2 were females. Thirty three (33) patients were known haemophilic and nine (9) cases were newly diagnosed during our study period. Most of the patients were below 20 years of age. 36 (81\%) patients were Factor VIII deficient which was most common. Out of these 36 patients, 17 had severe, 8 had moderate and 11 had mild factor VIII deficiency. Factor IX deficiency was seen in eight (8) patients which accounted for 17\% of cases. Five (5) cases had severe and three (3) cases had mild factor IX deficiency. One (1) case was Factor X deficient. There were 18 incidences of hospitalizations in our hospital of which 14 had factor VIII deficiency, 3 had factor IX deficiency and one had factor X deficiency. 87 Out Patient visits were recorded for haemophilia patients who were managed with factor replacement and/or other treatments as OPD basis. Hospitalizations were more in severe cases (58\%) than in others (27\%).35 cases presented with joint bleed, 11 cases with intramuscular bleed, one (1) case with life threatening intra-abdominal bleed, nine (9)cases with bleeds from teeth and gums and three (3)cases presented with epistaxis. Of the nine (9) newly diagnosed cases, three (3) presented with bleeding following dental procedures, one(1) with bleeding following surgery, two (2) with joint pain and 
deformity and three (3) were diagnosed after screening of siblings. All cases of major bleed received Factor and/or FFP replacement. One patient of severe factor XIII deficiency died after an intracranial bleeding. 22 patients with factor VIII and factor IX level below $<1 \%$ were regarded as severe factor deficient cases and were found to have 14 hospitalizations, multiple OPD visits and one (1) mortality. On the other hand 23 patients with their factor level in mild (5 to 40\%) and moderate (1 to 5\%) range had only 4 hospitalizations, infrequent OPD consultations and no mortality. Two females were found to have mild factor VIII deficiency.

Conclusion: We have presented the demographics of factor deficient patients coming to the eastern most Tertiary Care center of India over a period of one year. As per the estimated number of expected cases, we feel that Haemophilia is grossly under diagnosed in this region. Factor VIII was most common and most of the patients were below the age of 20 years. The patients with severe factor deficiency (Factor count <1\%) were more frequently hospitalized and had most of the complications. A prophylactic Factor replacement in severe factor deficiency cases and females would reduce the mortality and morbidity in these groups of patients to a great extent.

Keywords: Haemophilia A, Haemophilia B, Factor VIII, Factor IX, Factor Deficiency

\section{INTRODUCTION}

Haemophilia is an X-linked congenital bleeding disorder caused by coagulation factor deficiency that may present with bleeding episodes which may range from minor to severe life threatening. In most cases a definite family history in maternal side of the family is present but in upto $30 \%$ cases no family history is there. The suffering and risk of complications in these patients is high and a normal life is far from expected in untreated severe cases. Factor replacement is ideal treatment but very costly and affordable only with proper government or any society funding. Other ancillary managements like physiotherapy, physical rehabilitation of disability, life style and occupational adjustments and are also important in proper management of the condition. Prompt treatment of any acute medical emergencies in these patients is very important and usually requires special care in terms of blood and blood products.

\section{AIMS AND OBJECTIVES}

We studied all the patients with factor deficiency who came to Assam Medical College and Hospital, Dibrugarh for one year from $1 / 7 / 14$ to $31 / 6 / 15$ with respect to their clinical presentation, severity, treatment and outcome. Earlier when patients had to buy factors, many patients could not afford and we had to mostly rely on Fresh Frozen Plasma (FFP) and/or Blood transfusion. As factors arenow available in government medical college free of cost to haemophilia patients, we were also trying to identify a subgroup of patients who could benefit from prophylactic factor.

\section{MATERIALS AND METHODS}

All patients who attended the Department of Medicine and Paediatrics in a tertiary care Hospital which caters to Eastern most part of our country were studied. A detailed family history was taken and untested siblings were counselled and tested for factor deficiency. Factors were given to indicated patients with mild involvement as out-patients department (OPD) basis, but more severe cases were hospitalized. Proper clinical examination, routine blood tests and physiotherapy were advised in hospitalized patients.WFH Guidelines $\left(2^{\text {nd }}\right.$ Ed) was used as a standard treatment protocol. Significant Resource Constraint Guidelines (TABLE 7-2, Page 72 GUIDELINES FOR THE MANAGEMENT OF HAEMOPHILIA, $2^{\mathrm{ND}}$ ) was used to determine the amount of factor to be given to any particular patient. Patients with factor level from $5 \%$ to $40 \%$ were considered to have mild, from $>1 \%$ to $<5 \%$ were considered to be moderate and those $<1 \%$ were considered to be severe factor deficient patients.

\section{RESULTS AND OBSERVATIONS}

Forty five (45) cases were studied during this one year period of which, 43 were males and 2 were females. Thirty three (33) patients were known haemophilic and nine (9) cases were newly diagnosed during our study period. Most of the 
patients were in the age group of below 5 years (10), 11 to 15 years (12) and 16 to 20 years (10) (Figure no 1).

Figure 1 : Demographic profile

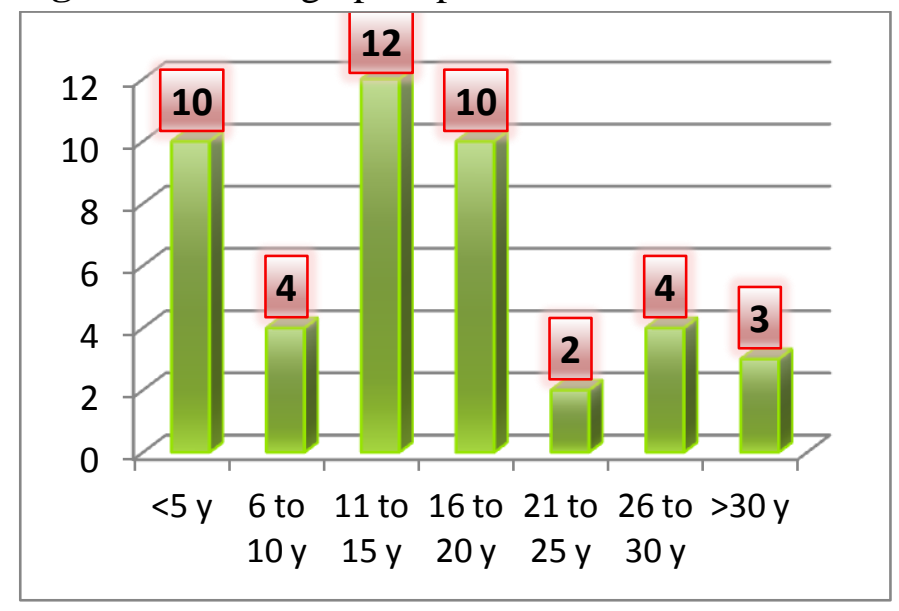

$36(81 \%)$ patients were Factor VIII deficient which was most common. Out of these 36 patients, 17 had severe, 8 had moderate and 11 had mild factor VIII deficiency. Factor IX deficiency was seen in eight (8) patients which accounted for $17 \%$ of cases. Five (5) cases had severe and three (3)cases had mild factor IX deficiency. One (1) case was Factor X deficient (Figure 2).

Figure 2 : Factor deficiency with severity

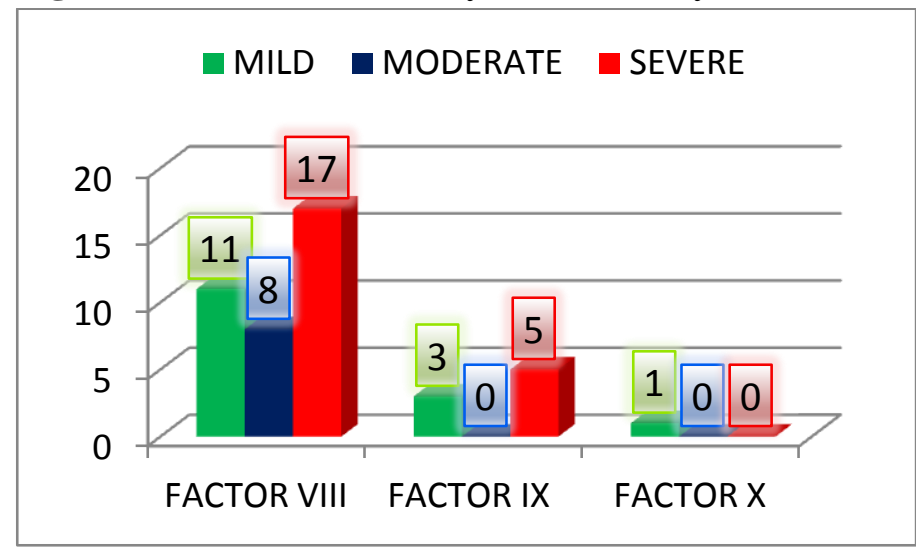

There were 18 incidences of hospitalizations in our hospital of which 14 had factor VIII deficiency, 3 had factor IX deficiency and onehad factor $\mathrm{X}$ deficiency. 87 Out Patient visits were recorded for haemophilia patients who were managed with factor replacement and/or other treatments as OPD basis. Hospitalizations were more in severe cases (58\%) than in others $(27 \%) .35$ cases presented with joint bleed, 11 cases with intramuscular bleed, one (1) case with life threatening intra-abdominal bleed, nine (9)cases with bleeds from teeth and gums and three (3)cases presented with epistaxis. Out of the 35 cases who presented with joint bleed 21 had knee joint involvement, six (6) had ankle joint involvement, four (4) had wrist involvement, five (5) had elbow involvement and two(2) had shoulder joint bleed. Out of the 11 cases of intramuscular bleed, seven (7) patients had psoas bleed, two (2) patients had bleed in biceps and one each had bleed in triceps and forearm. Of the nine (9) newly diagnosed cases, three (3) presented with bleeding following dental procedures, one(1) with bleeding following surgery, two (2) with joint pain and deformity and three (3) were diagnosed after screening of siblings (Figure 3). All cases of major bleed received Factor and/or FFP replacement. One patient of severe factor XIII deficiency died after an intracranial bleeding.

Figure 3: Clinical presentation of haemophilia patients

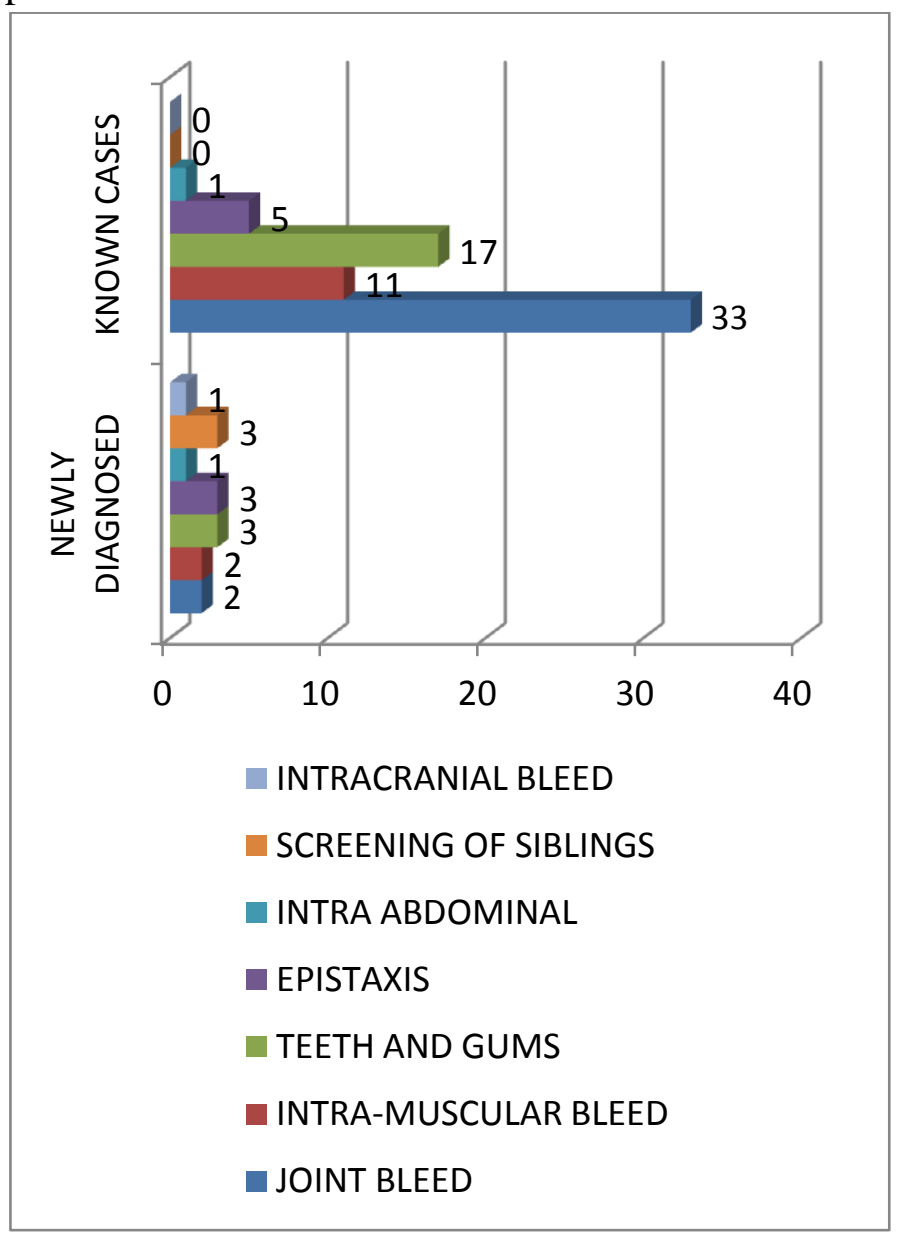


22 patients with factor VIII and factor IX level below $<1 \%$ were regarded as severe factor deficient cases and were found to have 14 hospitalizations, multiple OPD visits and one (1) mortality. On the other hand 23 patients with their factor level in mild (5 to $40 \%$ ) and moderate ( 1 to $5 \%$ ) range had only 4 hospitalizations, infrequent OPD consultations and no mortality. These clearly showed that severe factor deficiency $(<1 \%)$ cases had the worst morbidity and mortality.

Figure 4 : Hospitalization and mortality in severe haemophilia vs. Mild and moderate cases

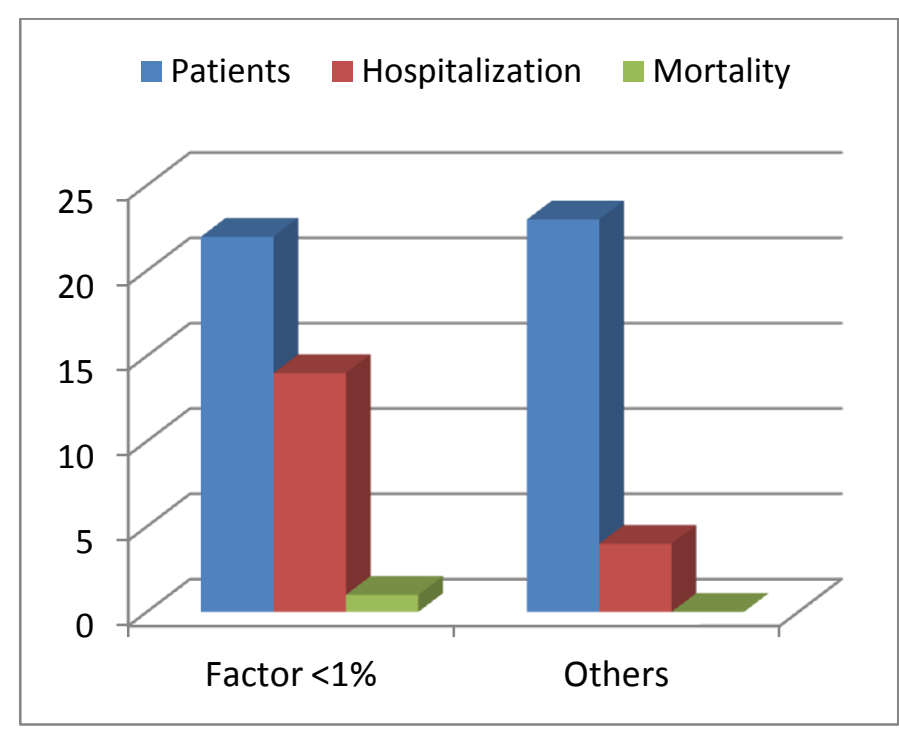

Two females were found to have mild factor VIII deficiency. Both of them came to us before menarche at the age of eight (8) and eleven (11) respectively. One of them had no family history of haemophilia and was accidently diagnosed when she had bleeding from tooth and gums. She was advised to undergo genetic testing in a higher centre. Another female had family history in her maternal side but no history of consanguineous marriage was elicited. Literature indicates that sometime a carrier may behave as a mild factor deficiency case.

\section{DISCUSSION}

Haemophilia has an incidence of 1 per 10,000 births, manifesting as spontaneous or traumainduced hemorrhagic episodes in patients, progressing to chronic disability and premature mortality in untreated patients or patients with suboptimal treatment ${ }^{1}$. There is estimated about 77,000 patients with haemophilia A and B in India ${ }^{1}$. Indian data are reported by the HFI. The data showed that in 2011, India reported 14,718 patients with bleeding disorders and 11,586 patients with haemophilia $\mathrm{A}^{2}$. India, the only developing country in the list of the top five countries with the highest number of reported haemophilia patients (USA, India, Brazil, China and UK) reports the third largest number of patients with bleeding disorders, and the second highest number of patients with haemophilia A.

Using the population data for 2011 from the Census of India ${ }^{3}$ and a prevalence of haemophilia A of 4 per $1,00,000$, the estimated number of haemophilia patients in India would be around 48,407. Thus, India may have over 70,000 patients with haemophilia $\mathrm{A}$ and B.Table 1 shows the observed number of cases (calculated at a prevalence of 0.9 per 1,00,000 population) and estimated number of patients (calculated at 4 per $1,00,000$ population) with haemophilia A in North East states of the India ${ }^{1}$.

Table 1: Expected number of patients with Factor deficiency from North East India (Kar Aet al , Epidemiology and social costs of haemophilia in India, Indian J Med Res 140, July 2014, pp 19-31)

\begin{tabular}{|l|l|l|l|}
\hline S1 no & State & $\begin{array}{l}\text { Observed } \\
\text { number of cases }\end{array}$ & $\begin{array}{l}\text { Expected } \\
\text { number of cases }\end{array}$ \\
\hline 1 & $\begin{array}{l}\text { Arunachal } \\
\text { Pradesh }\end{array}$ & 12 & 55 \\
\hline 2 & Assam & 280 & 1246 \\
\hline 3 & Manipur & 24 & 108 \\
\hline 4 & Meghalaya & 26 & 118 \\
\hline 5 & Mizoram & 10 & 43 \\
\hline 6 & Nagaland & 17 & 79 \\
\hline 7 & Sikkim & 2 & 24 \\
\hline 8 & Tripura & 33 & 146 \\
\hline
\end{tabular}

Our tertiary centre caters to eastern Assam, Eastern Arunachal Pradesh and some parts of Nagaland. As there is no published data on the demographics of haemophilia in this region, our study being the first 
of its kind from Eastern most tertiary care centre in India, aims to investigate and report the case we have encountered during the one year of study period. We had 45 documented cases with 36 Factor VIII deficiency, 7 factor IX deficiency and one factor $\mathrm{X}$ deficiency case. This emphasises the fact that haemophilia is hugely under diagnosed in this part of the country. This can be attributed to the lack of awareness among physicians as well as general population. We had a case which was being treated as a Juvenile Rheumatoid Arthritis for many years when actually he was a case of moderate Factor XIII deficiency with repeated joint bleeds and joint deformity.

Kar A et al studied 1467 patients and found the ratio of haemophilia A to haemophilia B patients was 4.2:1, with majority $(85 \%)$ being severe patients with FVIII deficiency ${ }^{4}$. We found the Haemophilia A to Haemophilia B ratio to be $4.5: 1$, with $80 \%$ being factor VIII deficient. Jayandharan $G$ et al analysed DNA from 109 unrelated Indian patients with Haemophilia A (HA) for their FVIII gene defects. Among these patients $89(82 \%)$ had severe (FVIII:C<1\%) HA, $11 \quad(10 \%)$ had moderate (FVIII:C 1-5\%) HA and nine (8\%) had mild (FVIII:C 5-30\%) HA ${ }^{5}$. We also found that out of 36 factor VIII deficient patients, $17(47.2 \%)$ had severe, $8(22.2 \%)$ had moderate and 11(30.6\%) had mild factor VIII deficiency.

Most of the patients were teenagers and children with very few patients above 25 years of age. Kar A et al also found that almost $77 \%$ of severe haemophilia A patients were under 20 years of age ${ }^{4}$. Most of them had a family history of haemophilia in maternal side of the family except 8 who did not give any significant family history of any bleeding disorders. Bhattacharya DK et al also noted that nearly one third of cases of haemophilia occur with no preceding family history, possibly from new genetic mutation ${ }^{6}$.

Joint bleed was the most common complication encountered in the patients with most common joint being the knee joint followed by the ankle, elbow, wrist and shoulder which correlates with most of the published data. Most of the patients with joint involvement were given factor in outpatients department and sent to home. Home management was also encouraged in most patients. 11 patients presented with intramuscular bleed with psoas being the most common followed by biceps, triceps and forearm. Most of these patients with intramuscular bleeds were hospitalized.

The most common minor bleed was from teeth and gums which mostly resolved with conservative treatment without factors except in 3 cases where bleeding did not stop after a dental procedure. They were hospitalized and treated with factor. Five cases of epistaxis were also treated conservatively.

One case of Sever factor XIII infant died after an intracranial bleed which was massive and included most of the frontal, partial and temporal lobes with intraventricular extension and midline shift. He died despite factor replacement.

One patient was found to have Factor X deficiency soon after birth and is of prophylactic fresh frozen plasma fortnightly. He has not had any major bleeding episodes except for few minor bruises.

Of the two females who presented with factor VIII deficiency, both had factor levels at mild level. One was actually a carrier as suggested by her family history but we found literature that sometimes a carrier may present as mild haemophilia. She presented before menarche with gum bleed after a dental procedure. In another case no family history was found and we have suspected a spontaneous mutation and suggested a genetic testing for her. Nair PS et $\mathrm{al}^{7}$ noted that females may exhibit a moderate to severe phenotypic expression of Haemophilia due to one of the following pathophysiology like (i) skewed inactivation of the $\mathrm{X}$ chromosome leading to predominant expression of the mutated allele as a result of a preferential inactivation of the $\mathrm{X}$ chromosome with the wildtype F8 gene, (ii) Turner syndrome, (iii) translocation or (iv) males with a female phenotype due to mutation in the sex-determining region $\mathrm{Y}$ (SRY) gene on the Y chromosome, i.e. Swyer syndrome combined with a mutation in the $F 8$ gene. Nair PS et al also noted that in India, as consanguineous marriages are very common in 
certain communities (up to $30 \%$ ), the likelihood of encountering female Haemophilia is higher.

\section{CONCLUSION}

We have presented the demographics of factor deficient patients coming to the eastern most Tertiary Care center of India over a period of one year. As per the estimated number of expected cases, we feel that Haemophilia is grossly under diagnosed in this region. Factor VIII was most common and most of the patients were below the age of 20 years. The patients with severe factor deficiency (Factor count <1\%) were more frequently hospitalized and had most of the complications. A prophylactic Factor replacement in severe factor deficiency cases and females would reduce the mortality and morbidity in these group of patients to a great extent.

\section{REFERENCE}

1. Kar A, Phadnis S, Dharmarajan S, Nakade J. Epidemiology and social costs of haemophilia in IndiaIndian J Med Res 140, July 2014, pp 19-3

2. World Federation of Haemophilia (WFH). Report on the Annual Global Survey 2011. Canada: WFH; 2013. Available from: http://www1.wfh.org/publications/files/pdf1488.pdf, accessed on May 9, 2013.

3. Census of India 2011, Office of the Registrar General and 15. Census Commissioner, India, Controller of Publications, New Delhi. Available from: http://www.censusindia.gov.in/2011-provresults/prov_results_paper1_india.html, accessed on October 12, 2012.

4. Kar A, Potnis-Lele M, Descriptive epidemiology of haemophilia in Maharashtra, India. Haemophilia, Nov 2001, Vol 7, Issur 6, 561-567

5. Jayandharan G, Shaji RV, Baidya S, Nair SC, Chandy M, Srivastava A,Identification of factor VIII gene mutations in 101 patients with haemophilia A: mutation analysis by inversion screening and multiplex PCR and CSGE and molecular modelling of 10 novel missense substitutions.Haemophilia. 2005 Sep;11(5):481-91.

6. Dilip K. Bhattacharya. Haemophilia in the Indian Scenario; Int J Hum Genet, 6(1): 3339 (2006)

7. Nair PS, Shetty S, and Ghosh K,A homozygous female Haemophilia A,Indian J Hum Genet. 2012 Jan-Apr; 18(1): 134-136. 